\title{
Efeitos de Dopamina e Noradrenalina no Fluxo Sangüíneo Regional no Tratamento do Choque Séptico*
}

\author{
Dopamine and Noradrenaline Effects in the Blood Flux \\ Regional on Therapeutic in the Septic Shock
}

\author{
Milena Penteado Ferraro Miranda', Francisco Garcia Soriano², Silvia Regina Secoli ${ }^{3}$
}

\section{RESUMO}

JUSTIFICATIVA E OBJETIVOS: A noradrenalina e a dopamina são utilizadas, no estado de choque, com o intuito de oferecer suporte hemodinâmico e restabelecer a perfusão tecidual. As ações farmacológicas desses vasopressores podem ser variadas, deste modo, o uso necessita, por parte do clinico, interpretação dos efeitos hemodinâmicos, com observação das variáveis sistêmica e regional. O objetivo deste estudo foi analisar as publicações acerca dos efeitos da noradrenalina e dopamina em baixas doses na perfusão hepatoesplâncnica e renal no tratamento do choque séptico.

MÉTODO: Foram selecionados artigos $(n=27)$ sobre o uso de noradrenalina e dopamina em choque séptico, publicados no período de 1997 a setembro de 2007 revisados na PubMed, base de dados da National Library of Medicine (NLM). Utilizou-se o recurso MESH com os descritores noradrenaline, dopamine e sepsis.

RESULTADOS: Os efeitos de dopamina e noradrena-

\footnotetext{
1. Enfermeira. Especialista em Enfermagem em Terapia Intensiva. Hospital Universitário da USP.

2. Professor Doutor da Disciplina de Emergências Clínicas do Departamento de Clínica Médica da FMUSP

3. Enfermeira. Professora Doutora do Departamento de Enfermagem Médico-Cirúrgica da Escola de Enfermagem da USP.
}

*Recebido da Faculdade de Medicina da Universidade de São Paulo (FMUSP), São Paulo, SP

Apresentado em 07 de novembro de 2007

Aceito para publicação em 26 de fevereiro de 2008

Endereço para correspondência:

Milena Penteado Ferraro Miranda

Rua Mesquita, 210

01544-010 São Paulo, SP

Fone: (11) 3586-0242

E-mail: enfi_mi@yahoo.com.br

(C)Associação de Medicina Intensiva Brasileira, 2008 lina na perfusão renal demonstraram ser semelhantes em relação ao aumento da diurese e não alteração na depuração de creatinina. A noradrenalina não afetou a perfusão tissular renal, apesar de elevar o tônus vascular. Quanto aos efeitos esplâncnicos, os dois fármacos demonstraram aumentar o fluxo sangüíneo, porém redistribuem o sangue nesse compartimento.

CONCLUSÕES: Os estudos analisados foram controversos não demonstrando consenso acerca do fármaco mais oportuno para o restabelecimento hemodinâmico e manutenção de perfusão regional adequada.

Unitermos: cuidados críticos, dopamina, norepinefrina, sepse.

\section{SUMMARY}

BACKGROUND AND OBJECTIVES: Norepinephrine and dopamine are used, in the state of shock, with the intention of offering hemodynamic support and to reestablish tissue perfusion. The pharmacological effects of these vasopressors can be diverse, for this reason, their use requires, through the clinician, an interpretation of the hemodynamic effects with observation of the systemic variations and region. With this in mind, the objective of this study was to analyze the publications regarding the effects of norepinephrine and lowdose dopamine in hepatosplenic perfusion and renal in treatment of septic shock.

METHODS: Articles were selected $(n=27)$ concerning the use of norepinephrine and dopamine in septic shock, published during the period of 1997 to September 2007, revised in PubMed, data base of the National Library of Medicine (NLM). The MESH method was utilized with the descriptors norepinephrine, dopamine and sepsis.

RESULTS: The effects of dopamine and norepinephrine in kidney perfusion are similar; there is an increase in diuresis and no change in creatinine clearance. No- 
repinephrine did not affect kidney tissue perfusion in spite of the increase of vascular tone. Regarding the splancnic effects, these drugs showed an increase in blood flow, though redistributing the blood in this compartment.

CONCLUSIONS: The best agent for the hemodynamic reestablishment that keeps the adequate regional perfusion remains inconclusive.

Key Words: critical care, dopamine, norepinephrine, sepsis.

\section{INTRODUÇÃO}

O estado de choque pode ser definido como o desequilíbrio entre a oferta e a demanda de oxigênio celular. Esta entidade é classificada de acordo com o padrão hemodinâmico em: cardiogênico, hipovolêmico, obstrutivo e séptico. Os três primeiros levam a redução do débito cardíaco (DC), hipoperfusão e metabolismo anaeróbio1. No choque séptico, ocorre liberação de mediadores pró-inflamatórios e formação de superóxidos, causando redução acentuada na resistência vascular periférica (RVP), evoluindo com aumento do $\mathrm{DC}^{2}$.

O choque séptico encontra-se associado a alterações cardiovasculares importantes, incluindo grave vasodilatação periférica - responsável pela hipotensão arterial, definida como pressão arterial média (PAM) $<60 \mathrm{mmHg}$ ou pressão arterial sistólica (PAS) $<90 \mathrm{mmHg}$ ou diminuição de $40 \mathrm{mmHg}$ na PAS de base, e alterações na distribuição do fluxo sanguíneo regional, especialmente nas regiões esplâncnica e renal ${ }^{3}$.

Para correção dessas alterações sistêmicas são utilizadas noradrenalina e dopamina, as quais podem modular a atividade metabólica, influenciar na distribuição do fluxo sangüíneo para vísceras e rins e no balanço entre oferta e demanda de oxigênio para vários órgãos. Todavia, esses efeitos podem ser influenciados por vários fatores, mas, sobretudo pela catecolamina e dose administrada ${ }^{1,3}$. Assim, o ajuste posológico desses agentes encontra-se condicionado aos efeitos hemodinâmicos e transporte de oxigênio.

No contexto atual há controvérsias acerca do uso dessas catecolaminas no que diz respeito aos efeitos farmacológicos, especialmente da dopamina em baixas doses.

Dopamina em baixas doses é definida como a dose que produz, preferencialmente, efeitos dopaminérgicos e $\beta$-adrenérgicos $(<5 \mu \mathrm{g} / \mathrm{kg} / \mathrm{min})$, por essa razão causa vasodilatação renal e esplâncnica em animais e humanos saudáveis ${ }^{4}$. Na clinica, durante muito tempo, esses efeitos sustentaram o uso dessa catecolamina, porém estudos recentes têm mostrado que o seu uso pode não oferecer benefício terapêutico adicional.

Tendo em vista essas considerações e que a falta de evidências, freqüentemente, favorece práticas impróprias, muitas vezes, baseadas em experiências individuais, o objetivo deste estudo foi analisar as publicações acerca dos efeitos da noradrenalina e da dopamina em baixas doses, na perfusão renal e hepatoesplâncnica, no tratamento do choque séptico.

\section{MÉTODO}

Trata-se de uma revisão de literatura realizada na PubMed, base de dados da National Library of Medicine (NLM). Para a busca de informações utilizou-se o recurso MESH com os descritores norepinephrine, dopamine e sepsis, cujo limite contemplou o período de 1997 a setembro de 2007. Para a seleção dos artigos adotaram-se os seguintes critérios de elegibilidade: possuir resumo, ter sido realizado em pacientes adultos ou em animais, ser estudo oriundo da prática clinica, de revisão, de ensaios clínicos, de metanálise, encontrarem-se disponíveis via on line ou em bibliotecas nacionais, e apresentar o tema dopamina e/ou noradrenalina relacionado ao uso no choque séptico.

Dentre os 45 artigos identificados 11 se repetiram durante as buscas com diferentes descritores, e a leitura mostrou que 18 apresentaram o tema objeto de analise desta revisão. Foram incluídos, outros nove artigos indicados por especialistas. Assim sendo, a amostra ficou constituída de 27 artigos.

\section{RESULTADOS}

Dentre os estudos analisados $62,9 \%(n=17)$ foram provenientes de ensaios clínicos realizados, $55,5 \%$ ( $n=$ 15) realizados com adultos em choque séptico, $33,3 \%$ $(n=9)$ investigaram a noradrenalina, 33,3\% ( $n=9)$ a dopamina, 33,3\% $(n=9)$ compararam as duas catecolaminas. A maioria $(54,2 \%)$ das publicações ocorreu no período de 2003 a 2006.

No que concerne aos objetivos verificou-se que $44,4 \%$ $(n=12)$ dos estudos analisaram os efeitos de dopamina/noradrenalina ou ambas na perfusão renal; 25,9\% ( $n=7)$ na hepatoesplâncnica; $14,8 \%(n=4)$ analisaram na perfusão renal e hepatoesplâncnica. Apenas 14,8\% $(n=4)$ dos artigos investigaram o impacto do uso desses fármacos vasopressores na mortalidade (Quadro 1) 
Quadro 1 - Características Gerais das Publicações

\begin{tabular}{|c|c|c|c|}
\hline Autores & Tipos de Estudos & Amostras & Objetivos do Estudos \\
\hline Reinelt e col. & Ensaio Clínico & $\begin{array}{l}\text { Adultos em choque séptico } \\
(\mathrm{n}=10)\end{array}$ & $\begin{array}{l}\text { Comparar noradrenalina e fenilefrina quanto aos parâmetros } \\
\text { hemodinâmicos esplâncnicos, oxigenação e metabólicos. }\end{array}$ \\
\hline Bellomo e col. & Experimental & $\begin{array}{l}\text { Cães em choque endotóxico in- } \\
\text { duzido }(n=9)\end{array}$ & $\begin{array}{l}\text { Avaliar o efeito de noradrenalina }(0,3 \mu \mathrm{g} / \mathrm{kg} / \mathrm{min}) \text { na perfusão } \\
\text { renal. }\end{array}$ \\
\hline Girbes e col. & Ensaio Clínico & $\begin{array}{l}\text { Adultos em choque séptico } \\
(\mathrm{n}=8)\end{array}$ & $\begin{array}{l}\text { Avaliar os efeitos de dopamina em doses dopaminérgica e } \\
\beta(9 \mu \mathrm{g} / \mathrm{kg} / \mathrm{min}) \text { associada à noradrenalina a } 0,29 \mu \mathrm{g} / \mathrm{kg} / \mathrm{min} \text {, } \\
\text { nas funções hemodinâmica, renal e neurohumoral. }\end{array}$ \\
\hline Ledoux e col. & Ensaio Clínico & $\begin{array}{l}\text { Adultos em choque séptico } \\
(\mathrm{n}=10)\end{array}$ & $\begin{array}{l}\text { Avaliar a perfusão regional com uso de noradrenalina após } \\
\text { aumento da PAM em } 65-75-85 \mathrm{mmHg} \text {. }\end{array}$ \\
\hline Day e col. & Ensaio Clínico & $\begin{array}{l}\text { Adultos em choque séptico e in- } \\
\text { fecção por malária grave } \\
(n=14)\end{array}$ & $\begin{array}{l}\text { Avaliar os efeitos da epinefrina e da dopamina em dose do- } \\
\text { paminérgica na função renal. }\end{array}$ \\
\hline Holmes e Walley & Revisão & $\begin{array}{l}\text { Artigos } \\
(\mathrm{n}=65)\end{array}$ & $\begin{array}{l}\text { Apresentar a melhor evidência e sumarizar os efeitos da in- } \\
\text { fusão de dopamina em dose dopaminérgica em pacientes } \\
\text { criticos. }\end{array}$ \\
\hline ANZICS* & Ensaio Clínico & $\begin{array}{l}\text { Adultos em estado crítico } \\
(\mathrm{n}=328)\end{array}$ & $\begin{array}{l}\text { Comparar os efeitos renais de dopamina em doses dopami- } \\
\text { nérgica e placebo. }\end{array}$ \\
\hline Chrusch e col. & Experimental & $\begin{array}{l}\text { Cães em choque séptico induzido } \\
(n=23)\end{array}$ & $\begin{array}{l}\text { Analisar os efeitos do tratamento com vasopressores (feni- } \\
\text { lefrina, dopamina, noradrenalina) e a associação de dobuta- } \\
\text { mina e noradrenalina na produção de lactato esplâncnico e } \\
\text { extração de lactato hepático. }\end{array}$ \\
\hline Martin e col. & Ensaio Clínico & $\begin{array}{l}\text { Adultos em choque séptico } \\
(\mathrm{n}=97)\end{array}$ & $\begin{array}{l}\text { Avaliar a influência dos efeitos da noradrenalina na mortali- } \\
\text { dade nos resultados do choque séptico. }\end{array}$ \\
\hline Kellum e Decker & Metanálise & $\begin{array}{l}\text { Artigos } \\
(\mathrm{n}=58)\end{array}$ & $\begin{array}{l}\text { Avaliar estudos sobre a incidência ou gravidade da insufi- } \\
\text { ciência renal aguda; necessidade de diálise e mortalidade } \\
\text { com uso de dopamina em dose dopaminérgica. }\end{array}$ \\
\hline Marik & $\begin{array}{l}\text { Revisão Sistemá- } \\
\text { tica }\end{array}$ & $\begin{array}{l}\text { Artigos } \\
(\mathrm{n}=15)\end{array}$ & $\begin{array}{l}\text { Determinar a magnitude do tratamento com dopamina em } \\
\text { dose dopaminérgica na função renal em pacientes com ris- } \\
\text { co ou com falência renal. }\end{array}$ \\
\hline Treggiari e col. & Experimental & $\begin{array}{l}\text { Suínos } \\
(n=14)\end{array}$ & $\begin{array}{l}\text { Avaliar os efeitos da noradrenalina, quanto a perfusão regio- } \\
\text { nal, após elevação pressórica em } 10 \mathrm{mmHg} \text { e } 20 \mathrm{mmHg} \text { do } \\
\text { valor basal ( } 52 \mathrm{mmHg} \text { ). }\end{array}$ \\
\hline Jakob e col. & Ensaio Clínico & $\begin{array}{l}\text { Adultos em choque séptico e após } \\
\text { cirurgia cardíaca. } \\
(n=20)\end{array}$ & $\begin{array}{l}\text { Analisar os efeitos de dopamina no fluxo sangüíneo sistê- } \\
\text { mico e regional. }\end{array}$ \\
\hline De Backer e col. & Ensaio Clínico & $\begin{array}{l}\text { Adultos em choque séptico } \\
(\mathrm{n}=20)\end{array}$ & $\begin{array}{l}\text { Avaliar e comparar os efeitos de dopamina, noradrenalina e } \\
\text { epinefrina na circulação esplâncnica. }\end{array}$ \\
\hline Verderese e col. & Experimental & $\begin{array}{l}\text { Cães em restrição hídrica. } \\
(\mathrm{n}=32)\end{array}$ & $\begin{array}{l}\text { Estudar o efeito da dopamina em dose dopaminérgica, da } \\
\text { solução hipertônica e da associação de ambas na função } \\
\text { renal. }\end{array}$ \\
\hline Nygren e col. & Ensaio Clínico & $\begin{array}{l}\text { Adultos em pós-operatório de ci- } \\
\text { rurgia cardíaca } \\
(n=18)\end{array}$ & $\begin{array}{l}\text { Observar os efeitos de noradrenalina e associação noradre- } \\
\text { nalina + dopamina, na perfusão esplâncnica após aumento } \\
\text { da pressão arterial em } 30 \% \text {, para atingir PAM de } 90 \mathrm{mmHg} \text {. }\end{array}$ \\
\hline $\begin{array}{l}\text { Giantomasso } \\
\text { col. }\end{array}$ & Experimental & $\begin{array}{l}\text { Ovelhas em choque endotóxico } \\
\text { induzido } \\
(n=7)\end{array}$ & $\begin{array}{l}\text { Comparar os efeitos renais e sistêmicos do uso de dopami- } \\
\text { na em dose dopaminérgica e média dose de noradrenalina } \\
(0,4 \mu \mathrm{g} / \mathrm{kg} / \mathrm{min}) \text {. }\end{array}$ \\
\hline Asfar e col. & Revisão & $\begin{array}{l}\text { Artigos } \\
(\mathrm{n}=20)\end{array}$ & $\begin{array}{l}\text { Analisar os estudos referentes aos efeitos esplâncnicos } \\
\text { e metabólicos da terapia vasopressora com dopamina, } \\
\text { noradrenalina, epinefrina, dobutamina usadas no choque } \\
\text { séptico. }\end{array}$ \\
\hline Debaveye e col. & Revisão & $\begin{array}{l}\text { Artigos } \\
(\mathrm{n}=41)\end{array}$ & $\begin{array}{l}\text { Analisar os estudos que utilizaram dopamina em dose do- } \\
\text { paminérgica e avaliaram os efeitos renais, esplâncnicos, } \\
\text { metabólicos e endócrinos. }\end{array}$ \\
\hline Guérin e col. & Ensaio Clínico & $\begin{array}{l}\text { Adultos em choque séptico } \\
(\mathrm{n}=12)\end{array}$ & $\begin{array}{l}\text { Comparar os efeitos sistêmicos de dopamina em dose } \beta \text { e } \\
\text { da noradrenalina }(0,1 \mu \mathrm{g} / \mathrm{kg} / \mathrm{min}) \text {. }\end{array}$ \\
\hline Albanese e col. & Ensaio Clínico & $\begin{array}{l}\text { Adultos em choque séptico } \\
(\mathrm{n}=20)\end{array}$ & $\begin{array}{l}\text { Comparar o efeito de noradrenalina }(0,3 \mu \mathrm{g} / \mathrm{kg} / \mathrm{min}) \text { no au- } \\
\text { mento da PAM de } 65 \text { para } 75 \mathrm{mmHg} \text {, a terlipressina nas } \\
\text { variáveis hemodinâmicas e função renal. }\end{array}$ \\
\hline
\end{tabular}


Quadro 1 - Características Gerais das Publicações - Continuação

\begin{tabular}{|c|c|c|c|}
\hline Autores & Tipos de Estudos & Amostras & Objetivos do Estudos \\
\hline Bourgoin e col. & Ensaio Clínico & $\begin{array}{l}\text { Adultos em choque séptico } \\
(\mathrm{n}=28)\end{array}$ & $\begin{array}{l}\text { Mensurar os efeitos de noradrenalina }(1,9 \mu \mathrm{g} / \mathrm{kg} / \mathrm{min}) \text { no au- } \\
\text { mento da PAM de } 65 \text { para } 85 \mathrm{mmHg} \text { nas variáveis de oxige- } \\
\text { nação e função renal. }\end{array}$ \\
\hline Oliveira e col. & Ensaio Clínico & $\begin{array}{l}\text { Idosos em choque séptico } \\
(n=67)\end{array}$ & $\begin{array}{l}\text { Analisar se o uso noradrenalina está associado a maior } \\
\text { ocorrência de mortalidade. }\end{array}$ \\
\hline Sakr e col. & $\begin{array}{l}\text { Ensaio Clínico } \\
\text { Multicêntrico }\end{array}$ & $\begin{array}{l}\text { Adultos em choque séptico e de } \\
\text { outras etiologias } \\
(n=1058)\end{array}$ & $\begin{array}{l}\text { Investigar a influência na mortalidade com o uso de dopa- } \\
\text { mina nas doses de }<3 \mu \mathrm{g} / \mathrm{kg} / \mathrm{min}, 3-5 \mu \mathrm{g} / \mathrm{kg} / \mathrm{min},>5 \mu \mathrm{g} / \\
\mathrm{kg} / \mathrm{min} \text {. }\end{array}$ \\
\hline Schmoelz e col. & Ensaio Clínico & $\begin{array}{l}\text { Adultos em choque séptico } \\
(\mathrm{n}=61)\end{array}$ & $\begin{array}{l}\text { Avaliar os efeitos de dopamina em dose dopaminérgica as- } \\
\text { sociada à noradrenalina }(0,5 \mu \mathrm{g} / \mathrm{kg} / \mathrm{min}) \text { em pacientes com } \\
\text { choque séptico com insuficiência renal aguda. }\end{array}$ \\
\hline Lauzier e col. & Ensaio Clínico & $\begin{array}{l}\text { Adultos em choque séptico } \\
(\mathrm{n}=23)\end{array}$ & $\begin{array}{l}\text { Comparar os efeitos de vasopressina e noradrenalina } \\
(0,1-2,8 \mu \mathrm{g} / \mathrm{kg} / \mathrm{min}) \text { para o aumento da PAM acima de } 70 \\
\text { mmHg, quanto às variáveis hemodinâmicas, disfunção de } \\
\text { órgãos e na ocorrência de eventos adversos. }\end{array}$ \\
\hline Hernandez e col & $\begin{array}{l}\text { Ensaio Clinico } \\
\text { Multicêntrico }\end{array}$ & $\begin{array}{l}\text { Adultos em choque séptico } \\
(\mathrm{n}=45)\end{array}$ & $\begin{array}{l}\text { Comparar noradrenalina ( }>0,1 \mu \mathrm{g} / \mathrm{kg} / \mathrm{min} \text { ) e dopamina ( }>10 \\
\mu \mathrm{g} / \mathrm{kg} / \mathrm{min} \text { ) na estabilização de pacientes em choques sép- } \\
\text { tico moderado e grave. }\end{array}$ \\
\hline
\end{tabular}

*Australian and New Zealand Intensive Care Society (ANZICS) Clinical Trials Group

\section{DISCUSSÃO}

Na clinica, a dopamina em dose dopaminérgica foi por muito tempo utilizada no intuito de prevenir a insuficiência renal aguda (IRA) ${ }^{5}$. O efeito natriurético desta catecolamina encontra-se intimamente relacionado à concentração plasmática de renina, ou seja, depende do balanço entre a vasodilatação renal estimulada pela dopamina e a vasoconstrição renal mediada pelo sistema renina-angiotensina-aldosterona ${ }^{6}$. Entretanto, a melhora da função renal não se encontra condicionada ao aumento do débito urinário (DU). Pelo contrário, o aumento do transporte de solutos para as células tubulares distais, produzido pelo efeito natriurético de dopamina aumenta o consumo de oxigênio medular,6. Nos adultos em choque séptico, a dopamina em baixas doses não reduziu a incidência de IRA ${ }^{5,7}$. Naqueles com risco ou falência renal esta catecolamina, também, não apresentou efeito renoprotetor ${ }^{8}$. Todavia observou-se que ela foi capaz de aumentar o fluxo sangüíneo renal ${ }^{6,9,10}$ aumentando o DU ${ }^{5,7,9,11}$, mas sem alterar a depuração da creatinina, a creatinina plasmática, os níveis de renina e a concentração de aldosterona no plasma ${ }^{6,8,10}$.

Os achados experimentais apontaram que o uso da dopamina em dose dopaminérgica associada à solução hipertônica infundida em cães causou aumento do fluxo plasmático efetivo renal e da diurese/natriurese apenas nos animais hidratados, não ocorrendo naqueles submetidos à infusão de dose dopaminérgica em condição de déficit de volume. Nestes animais observou-se apenas diminuição da resistência vascular renal (RVR) ${ }^{9}$.

De modo geral, o uso da dopamina em baixas doses utilizados em pacientes criticamente enfermos sob o risco de falência renal não conferiu efeito clinicamente significante como protetor renal ${ }^{5-8,10}$. Além disso, esta catecolamina, devido sua ação diurética, pode apresentar efeitos deletérios em pacientes normo ou hipovolêmicos ${ }^{5}$.

A dose ideal de noradrenalina deve restaurar a perfusão tissular sem causar vasoconstrição excessiva. Deste modo, deve-se usar infusões até $0,5 \mu \mathrm{g} / \mathrm{kg} / \mathrm{min}$ e a manutenção da PAM entre $65-70 \mathrm{mmHg}^{2,3}$.

Estudos experimentais com noradrenalina apontaram que quando a PAM elevou-se de 52 para $65 \mathrm{mmHg}$ houve melhora no fluxo sangüíneo renal ${ }^{12}$. Este tipo de resposta é esperado, visto que o valor pressórico médio de $60 \mathrm{mmHg}$ representa o mínimo necessário para a auto-regulação do fluxo sanguíneo renal, coronariano e cerebral.

O aumento do fluxo sanguíneo renal promovido pela noradrenalina não resulta apenas da elevação da pressão de perfusão renal ${ }^{13}$. Sua ação na resistência arteriolar eferente normaliza a RVR aumentando, assim, a fração de filtração. Além disso, essa elevação, também, pode ser explicada pela redução da liberação do hormônio antidiurético ${ }^{14}$.

$\mathrm{O}$ aumento da PAM para valores acima de $70 \mathrm{mmHg}$ não alterou o fluxo sanguíneo renal, apesar de causar aumento significativo da RVR ${ }^{15}$. Identificou-se apenas um 
estudo em que, mesmo com PAM acima de $70 \mathrm{mmHg}$, observou-se aumento no DU e melhora na depuração da creatinina ${ }^{16}$. Todavia cabe destacar que neste estudo os pacientes encontravam-se na fase hiperdinâmica do choque, que apesar de haver redução da resistência vascular sistêmica, o DU mostrava-se normal.

Outros estudos não observaram alteração no DU ou depuração de creatinina ${ }^{17,18}$.

$\mathrm{Na}$ comparação da monoterapia com dopamina em baixas doses e noradrenalina $(0,4 \mu \mathrm{g} / \mathrm{kg} / \mathrm{min})$ verificouse que ocorreu aumento do fluxo sangüíneo renal e DU em ambos os tratamentos, todavia o efeito de noradrenalina foi mais pronunciado por aumentar o fluxo sanguíneo renal em $29 \%$, enquanto com dopamina esse aumento foi de $20 \%$. Nenhuma delas alterou a depuração de creatinina ${ }^{19}$.

O tratamento combinado de noradrenalina $(0,5 \mu \mathrm{g} / \mathrm{kg} /$ min) e dopamina em dose dopaminérgica em pacientes com IRA mostrou que houve aumento no DU e na depuração da creatinina ${ }^{20}$.

Contudo, na associação de dopamina em dose dopaminérgica e em dose $\beta$ (baixas doses) e noradrenalina $(0,29$ $\mu \mathrm{g} / \mathrm{kg} / \mathrm{min}$ ) os autores observaram aumento no DU e natriurese, mas não verificaram alteração na depuração da creatinina, concluindo que o tratamento não foi efetivo na melhora da função renal21 (Quadro 2).
Nos pacientes críticos como, por exemplo, os sépticos há prejuízo na capacidade de extração do oxigênio. Decorrente disso, o consumo torna-se dependente da oferta de oxigênio induzida pela manipulação terapêutica ${ }^{1,6}$.

O uso da dopamina em baixas doses em pacientes em choque séptico e em adultos submetidos à cirurgia cardíaca mostrou aumento no fluxo sanguíneo hepatoesplâncnico, porém diminuição do consumo de oxigênio, indicando redistribuição do fluxo $0^{5,6,22}$. Acredita-se que a dopamina modifique o estado redox celular por inibição direta do complexo enzimático do citocromo P450 ou por reduzir a atividade da NADH desidrogenase, acarretando disfunção celular hepatoesplâncnica ${ }^{22}$.

A noradrenalina utilizada para a manutenção da PAM em $70 \mathrm{mmHg}$ aumentou o fluxo sangüíneo esplâncnico, sem alterar o consumo de oxigênio ${ }^{23}$. Todavia, quando a PAM foi elevada para 65, 75 e $85 \mathrm{mmHg}$ não foram observadas alterações na perfusão regional esplâncnica, as quais foram analisadas através do gradiente entre $\mathrm{pCO}_{2}$ arterial e $\mathrm{pCO}_{2}$ gástrico. $\mathrm{O}$ aumento da PAM para $85 \mathrm{mmHg}$ não mostrou redistribuição do sangue na região esplâncnica ${ }^{15}$.

Estudo experimental mostrou que noradrenalina aumentou a oferta, o consumo de oxigênio esplâncnico e o fluxo sangüíneo microvascular jejunal, quando a PAM foi eleva-

Quadro 2 - Efeitos das Catecolaminas Dopamina e Noradrenalina na Função Renal Segundo Desfecho no Débito Urinário, Fluxo Sanguíneo Renal e Depuração de Creatinina.

\begin{tabular}{|c|c|c|c|c|}
\hline Catecolaminas & Débito Urinário & Fluxo Sanguíneo Renal & $\begin{array}{l}\text { Depuração de } \\
\text { Creatinina }\end{array}$ & Autores \\
\hline Noradrenalina & Não avaliado & Aumentado & Não avaliado & Bellomo e col. \\
\hline $\begin{array}{l}\text { Dopamina }+ \\
\text { Noradrenalina }\end{array}$ & Aumentado & Não avaliado & Inalterado & Girbes e col. \\
\hline Noradrenalina & Inalterado & Inalterado & Não avaliado & Ledoux e col. \\
\hline Dopamina & Inalterado & $\begin{array}{l}\text { Aumento }(<5 \mu \mathrm{g} / \mathrm{kg} / \mathrm{min}) \\
\text { Diminuído }(>10 \mu \mathrm{g} / \mathrm{kg} / \mathrm{min})\end{array}$ & Inalterado & Day e col. \\
\hline Dopamina & Não avaliado & Aumentado & Não avaliado & Holmes e Walley \\
\hline Dopamina & Aumentado & Não avaliado & Não avaliado & ANZICS * \\
\hline Dopamina & Aumentado & Não avaliado & Não avaliado & Kellum e Decker \\
\hline Dopamina & Não avaliado & Não avaliado & Não Avaliado & Marik \\
\hline Noradrenalina & Não avaliado & $\begin{array}{l}\text { Aumento (Aumento da PAM em } 10 \mathrm{mmHg} \text { ) } \\
\text { Inalterado (Aumento da PAM em } 20 \mathrm{mmHg} \text { ) }\end{array}$ & Não avaliado & Treggiari e col. \\
\hline Dopamina & Aumentado & Aumentado & Não avaliado & Verderese e col. \\
\hline $\begin{array}{l}\text { Dopamina } X \\
\text { Noradrenalina }\end{array}$ & $\begin{array}{l}\text { Aumentado (Dopamina) } \\
\text { Aumentado (Noradrenalina) }\end{array}$ & Aumentado & $\begin{array}{l}\text { Inalterado } \\
\text { Inalterado }\end{array}$ & Giantomasso e col. \\
\hline Dopamina & Aumentado & Não avaliado & Não avaliado & Debaveye e col. \\
\hline Noradrenalina & Aumentado & Não avaliado & Aumentado & Albanese e col. \\
\hline Noradrenalina & Inalterado & Não avaliado & Inalterado & Bourgoin e col. \\
\hline $\begin{array}{l}\text { Noradrenalina }+ \\
\text { Dopamina }\end{array}$ & Aumentado & Não avaliado & Elevado & Schmoelz e col. \\
\hline Noradrenalina & Inalterado & Não avaliado & Inalterado & Lauzier e col. \\
\hline
\end{tabular}

*Australian and New Zealand Intensive Care Society (ANZICS) Clinical Trials Group 
da em $10 \mathrm{mmHg}$. Entretanto, quando a PAM foi aumentada em $20 \mathrm{mmHg}$, para atingir $72 \mathrm{mmHg}$, a despeito do fluxo sangüíneo jejunal estar elevado, a extração de oxigênio e o consumo esplâncnico foram reduzidos. Assim, a noradrenalina parece alterar a distribuição do fluxo sangüíneo regional de modo inadequado, contribuindo para o desenvolvimento de falência de múltiplos órgãos ${ }^{13}$. Houve redução do fluxo sangüíneo esplâncnico quando a noradrenalina foi substituída pela fenilefrina - catecolamina sintética que atua quase exclusivamente em receptores $\alpha$ - retornando ao basal no momento em que a catecolamina natural foi re-introduzida ${ }^{23}$. Esta alteração pode ser explicada pelo fato da expressão dos receptores adrenérgicos diferir entre os leitos vasculares, e o esplâncnico $(\alpha)$, ao contrário do músculo esquelético $\left(\beta_{2}\right)$, ser particularmente responsivo à noradrenalina, especialmente com o aumento da infusão. Neste contexto, o aumento da estimulação desses receptores inicia excessiva vasoconstrição e redistribuição do fluxo sangüíneo na circulação esplâncnica'.

$\mathrm{Na}$ comparação de noradrenalina $(0,1 \mu \mathrm{g} / \mathrm{kg} / \mathrm{min})$ e dopamina em dose $\beta$ e em dose $\alpha$ os achados foram controversos. Quando as catecolaminas foram utilizadas para elevar a PAM até $80 \mathrm{mmHg}$ os resultados foram similares quanto ao aumento do fluxo sangüíneo hepatoesplâncnico. Não houve diferença entre elas no que se refere à oferta e o consumo de oxigênio nessa região. Porém, para a manutenção da PAM, da perfusão e da oxigenação hepatoesplâncnica com o uso da dopamina em dose $\beta$ ocorreu aumento do débito cardíaco, o qual foi responsável por elevar a demanda de oxigênio global. Em adição, esta catecolamina de- teriorou o balanço energético hepático ${ }^{24}$. Quando os valores pressóricos foram mantidos acima de $65 \mathrm{mmHg}$, os resultados mostraram-se similares quanto ao fluxo sangüíneo e a oferta de oxigênio esplâncnico. Entretanto, a dopamina em dose $\alpha$ foi associada à menor gradiente de saturação venosa de oxigênio mista hepático, indicando melhor balanço de oxigênio. A concentração de lactato e pH arterial foram similares, mas a concentração de glicose sérica foi maior com noradrenalina ${ }^{25}$.

Em estudo experimental observou-se que somente a dopamina em dose $\alpha$ aumentou o fluxo sangüíneo hepatoesplâncnico. $O$ consumo de oxigênio nessa região permaneceu inalterado com as duas catecolaminas ${ }^{26}$. $\mathrm{Na}$ análise dos efeitos da noradrenalina e noradrenalina associada à dopamina em baixas doses na circulação esplâncnica, verificou-se que a monoterapia aumentou significativamente $(p<0,05)$ a extração de oxigênio esplâncnico em pacientes com baixo índice cardíaco, sugerindo menor fluxo sangüíneo nessa região. A perfusão da mucosa jejunal e o $\mathrm{pCO}_{2}$ arterial gástrico não foram afetados quando se utilizou somente a noradrenalina. Porém, na combinação da dopamina em dose dopaminérgica e noradrenalina observou-se que a extração de oxigênio esplâncnico foi menor, a perfusão da mucosa jejunal foi maior e o $\mathrm{pCO}_{2}$ arterial gástrico não se alterou ${ }^{27}$.

Num estudo de revisão, os resultados quanto ao fluxo e consumo de oxigênio, $\mathrm{pH}$ intramucoso e $\mathrm{pCO}_{2}$ na região hepatoesplâncnica mostraram-se controversos, deste modo, não sendo possível os autores apresentarem uma conclusão definitiva acerca das diferenças entre as catecolaminas investigadas ${ }^{28}$ (Quadro 3).

Quadro 3 - Efeitos das Catecolaminas Dopamina e Noradrenalina no Fluxo Sangüíneo e Relação Oferta e Oxigênio Hepatoesplâncnico.

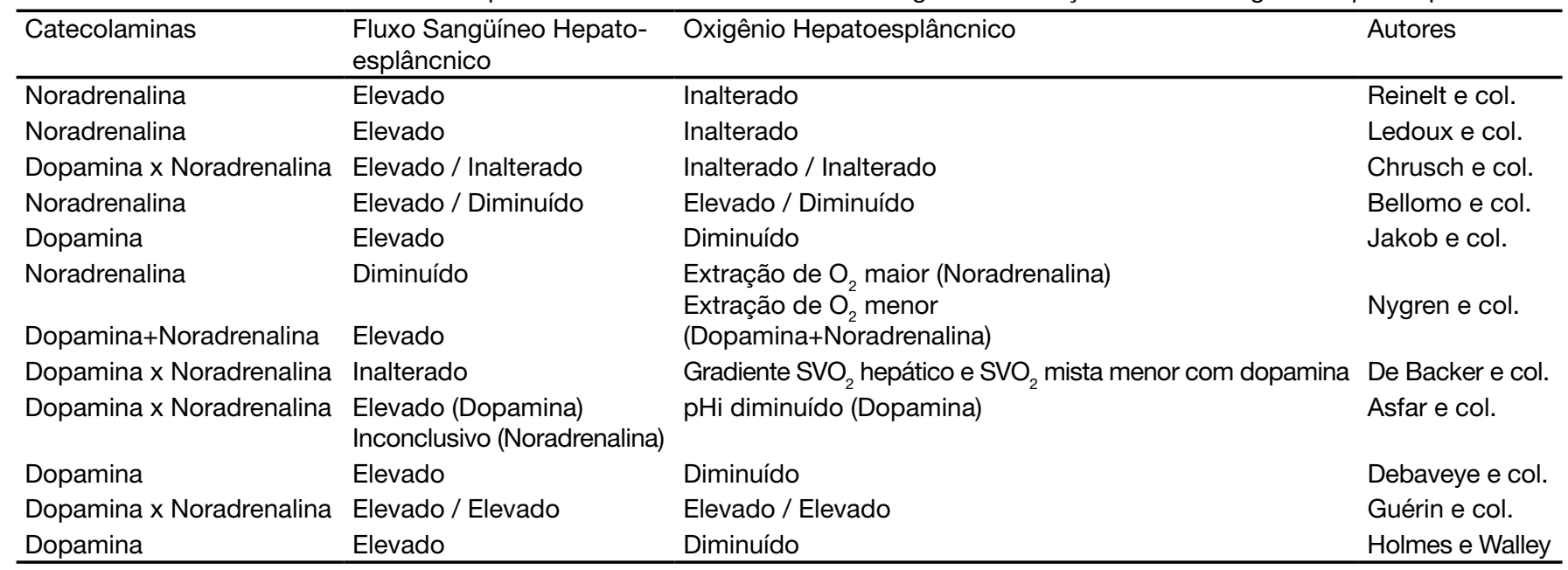

$\mathrm{SVO}_{2}$ - Saturação venosa de oxigênio

$\mathrm{pHi}-\mathrm{pH}$ intramucoso 
O impacto do uso da dopamina e da noradrenalina na ocorrência de desfechos negativos foi avaliada em alguns estudos.

Em estudo multicêntrico recente os autores investigaram o uso da noradrenalina $(>0,1 \mu \mathrm{g} / \mathrm{kg} / \mathrm{min})$ e dopamina ( $>10 \mu \mathrm{g} / \mathrm{kg} / \mathrm{min}$ ) e observaram que reações adversas como fibrilação atrial e taquicardia paroxística supraventricular foram mais freqüentes e mais graves no grupo submetido à tratamento com dopamina ${ }^{29}$.

Observou-se aumento na mortalidade em pacientes submetidos à tratamento com dopamina em doses $<3 \mu \mathrm{g} / \mathrm{kg} / \mathrm{min}$, 3-5 $\mu \mathrm{g} / \mathrm{kg} / \mathrm{min}$ e $>5 \mu \mathrm{g} / \mathrm{kg} / \mathrm{min}$, cujos fatores associados foram taquidisritmia e imunossupressão ${ }^{30}$.

Nos pacientes que receberam noradrenalina em doses superiores a $0,5 \mu \mathrm{g} / \mathrm{kg} / \mathrm{min}$ e naqueles que apresentavam falências prévias em dois ou mais órgãos, verificou-se aumento da mortalidade ${ }^{2}$. Nesta situação é difícil concluir que noradrenalina tenha contribuído no aumento desse evento, pois os pacientes apresentavam quadro clínico mais grave.

Na metanálise, cuja mortalidade foi avaliada em 11 estudos, observou-se que este desfecho foi similar entre os pacientes do grupo controle e do grupo com dopamina ${ }^{7}$.

Na comparação do tratamento com noradrenalina $(0,5$ a $5 \mu \mathrm{g} / \mathrm{kg} / \mathrm{min}$ ) e dopamina (16 a $25 \mu \mathrm{g} / \mathrm{kg} / \mathrm{min}$ ) em pacientes com persistente hipotensão, oligúria e acidose láctica, os autores observaram menor taxa de mortalidade com a noradrenalina, concluindo que esta catecolamina apresenta efeitos benéficos no manuseio hemodinâmico do choque séptico ${ }^{16}$.

\section{CONCLUSÃO}

A despeito de haver muitos estudos que avaliaram variáveis distintas, dificultando a comparação e a extração de conclusões definitivas, o presente estudo permitiu afirmar que os efeitos de dopamina e noradrenalina foram capazes de aumentar a pressão de perfusão renal e, conseqüentemente, o fluxo sanguíneo renal, elevando o DU em pacientes em choque séptico, porém, não demonstraram alteração na depuração de creatinina - um importante indicador da função renal. De modo geral, parece haver ineficácia do uso da dopamina em dose dopaminérgica para proteção ou tratamento da falência renal em pacientes críticos. Seu efeito natriurético acarreta aumento do consumo de oxigênio medular, podendo causar isquemia do órgão. Além disso, pode apresentar efeitos deletérios em pacientes normo ou hipovolêmicos, decorrente da sua ação diurética. A noradrenalina foi efetiva no restabelecimento da PAM, e capaz de manter inalterado o fluxo sanguíneo renal com PAM acima de $70 \mathrm{mmHg}$, demonstrando que não necessariamente ocorre vasoconstrição excessiva renal com esses níveis pressóricos. Quanto aos efeitos das catecolaminas no fluxo sanguíneo da região hepatoesplâncnica, os achados foram inconclusivos, não permitindo apontar o melhor agente para o restabelecimento hemodinâmico, com manutenção de adequada perfusão regional. Apesar de serem poucos os estudos, a dopamina apresentou maior numero de desfechos negativos como mortalidade e reações adversas, sendo apontadas as taquidisritmias como os principais fatores associados. Assim sendo, há um terreno fértil para a elaboração de ensaios clínicos, que apresentem rigor no método e utilizem variáveis que possibilitem comparações, no intuito de responder com dados mais conclusivos algumas controvérsias apontadas nesta e em outras revisões.

\section{REFERÊNCIAS}

01. Machado FS, Barreto AJ, Silva E - Classificação dos Diferentes Estados de Choque, em: Knobel E - Terapia Intensiva-Hemodinâmica. São Paulo, Atheneu; 2005;167-185.

02. Oliveira GM, Godoy $\mathrm{PH}$, Luiz RR et al - Influência da noradrenalina na mortalidade dos idosos com choque séptico. SOCERJ, 2005;18:254-260.

03. De Backer D, Vincent $\mathrm{JL}$ - Norepinephrine administration in septic shock: how much is enough? Crit Care Med, 2002;30:1398-1399.

04. Dório V, El Allaf D, Juchmes J et al - The use of low-dose dopamine in intensive care medicine. Arch Int Physiol Biochim Biophys 1984:92:(Suppl):S1-S20.

05. Debaveye YA, Van den Berghe GH - Is there still a place for dopamine in the modern intensive care unit? Anesth Analg, 2004;98:461-468.

06. Holmes CL, Walley KR - Bad medicine low-dose dopamine in ICU. Chest, 2003;123:1266-1275.

07. Kellum JA, M Decker $\mathrm{J}$ - Use of dopamine in acute renal failure: a metaanalysis. Crit Care Med, 2001;29:1526-1531.

08. Marik PE - Low-dose dopamine: a systematic review. Intensive Care Med, 2002;28:877-883.

09. Verderese MAL, Vianna PTG, Ganem EM et al - Efeitos renais e cardiovasculares da infusão de dopamina e da solução de cloreto de sódio a 7.5\%. Estudo experimental em cães com restrição hídrica. Rev Bras Anestesiol, 2003;53:600-609.

10. Day NP, Phu NH, Mai NT et al - Effects of dopamine and epinephrine infusions on renal hemodynamics in severe malaria and severe sepsis. Crit Care Med, 2000;28:1353-1362.

11. Australian and Zealand Intensive Care Society (ANZICS) Clinical Trials Group. Low-dose dopamine in patients with early renal dysfunction: a placebo-controlled randomized trial. Lancet, 2000;356:2139-2143.

12. Treggiari MM, Romand JA, Burgener D et al - Effects of increasing norepinephrine dosage on regional blood flow in a porcine model of endotoxin shock. Crit Care Med, 2002;30:1334-1339.

13. Bellomo R, Kellum JA, Wisniewski SR et al - Effects of norepinephrine on the renal vasculature in normal and endotoxemic dogs. Am J Respir Crit Care Med, 1999;159:1186-1192.

14. Martin C, Viviand $\mathrm{X}$, Leone $\mathrm{M}$ et al - Effect of norepinephrine on the outcome of septic shock. Crit Care Med, 2000;28:2758-2765.

15. LeDoux D, Astiz ME, Carpati CM et al - Effects of perfusion pressure on tissue perfusion in septic shock. Crit Care Med, 2000;28:2729-2732. 
16. Albanese J, Leone M, Delmas A et al - Terlipressin or norepinephrine in hyperdynamic septic shock: a prospective, randomized study. Crit Care Med, 2005;33:1897-1902.

17. Bourgoin A, Leone M, Delmas A et al - Increasing mean arterial pressure in patients with septic shock: effects on oxygen variables and renal function. Crit Care Med, 2005;33:780-786.

18. Lauzier F, Levy B, Lamarre P et al - Vasopressin or norepinephrine in early hyperdynamic septic shock: a randomized clinical trial. Intensive Care Med, 2006;32:1782-1789.

19. Di Giantomasso D, Morimatsu H, May $\mathrm{C}$ et al - Increasing renal blood flow: low-dose dopamine or medium-dose norepinephrine. Chest, 2004;125:2260-2267.

20. Schmoelz M, Schelling G, Dunker M et al - Comparison of systemic and renal effects of dopexamine and dopamine in norepinephrine-treated septic shock. J Cardiothorac Vasc Anesth, 2006;20:173-178.

21. Girbes AR, Patten MT, McCloskey BV et al - The renal and neurohumoral effects of the addition of low-dose dopamine in septic critically ill patients. Intensive Care Med, 2000;26:1685-1689.

22. Jakob SM, Ruokonen E, Takala J - Effects of dopamine on systemic and regional blood flow and metabolism in septic and cardiac surgery patients. Shock, 2002;18:8-13.

23. Reinelt $\mathrm{H}$, Radermacher $\mathrm{P}$, Kiefer $\mathrm{P}$ et al - Impact of exogenous (beta)adrenergic receptor stimulation on hepatosplanchnic oxygen kinetics and metabolic activity in septic shock. Crit Care Med, 1999;27:325-331.

24. Guerin JP, Levraut J, Samat-Long C et al - Effects of dopamine and norepinephrine on systemic and hepatosplanchnic hemodynamics, oxygen exchange, and energy balance in vasoplegic septic patients. Shock, 2005;23:18-24.

25. De Backer D, Creteur J, Silva E et al - Effects of dopamine, norepinephrine and epinephrine on the splanchnic circulation in septic shock: which is best? Crit Care Med, 2003;31:1659-1667.

26. Chrusch C, Bands C, Bose D et al - Impaired hepatic extraction and increased splanchnic production contribute to lactic acidosis in canine sepsis. Am J Respir Crit Care Med, 2000;161:517-526.

27. Nygren A, Thoren A, Ricksten SE - Effects of norepinephrine alone and norepinephrine plus dopamine on human intestinal mucosal perfusion Intensive Care Med, 2003;29:1322-1328.

28. Asfar P, De Backer D, Meier-Hellmann A et al - Clinical review: influence of vasoactive and other therapies on intestinal and hepatic circulations in patients with septic shock. Crit Care, 2004;8:170-179.

29. Hernandez G, Bruhn A, Larrondo F et al - DONORS: A Randomized, double blind controlled trial (RCT) comparing dopamine vs norepinephrine in moderate to severe septic shock. Intensive Care Medicine, 2007;33(Suppl2):S36.

30. Sakr Y, Reinhart K, Vincent JL et al - Does dopamine administration in shock influence outcome? Results of the Sepsis Occurrence in Acutely ill Patients (SOAP) Study. Crit Care Med, 2006;34:589-597. 